\title{
Synthesis of Cyclometallated Pt(II) Complexes of a Bulky Bipyridine Ligand
}

\section{Sarath D. Perera*}

Department of Chemistry, The Open University of Sri Lanka, Sri Lanka

\section{Abstract}

Treatment of 3,4,5,6-tetraphenyl-2,2'-bipyridine (LH) (7) with $\left[\mathrm{PtCl}_{2}(\mathrm{dmso})_{2}\right]$ (dmso = dimethyl sulfoxide) in chloroform afforded the cyclometallated square-planar platinum(II) complex $[\mathrm{PtCl}(\mathrm{L})]$ (8) and the octahedral $\mathrm{Pt}(\mathrm{IV})$ complex mer-[PtCl $3(\mathrm{~L})]$ (9) containing an anionic tridentate $\left(\mathrm{C}^{\wedge} \mathrm{N}^{\wedge} \mathrm{N}\right)$ ligand. The chloride of $(\mathbf{8})$ can be easily replaced by trifluoroacetate to yield [(L) $\left.\mathrm{PtO}_{2} \mathrm{CCF}_{3}\right]$ (10). Reaction of (8) with the alkyne $\mathrm{HC} \equiv \mathrm{CC}_{6} \mathrm{H}_{4} \mathrm{Bu}^{\mathrm{t}}-4$ resulted in the formation of $\left[(\mathrm{L}) \mathrm{PtC} \equiv \mathrm{CC}_{6} \mathrm{H}_{4} \mathrm{Bu}^{\mathrm{t}-4}\right]$ (11). Treatment of (8) with 4-dimetylaminopyridine (DMAP) and $\mathrm{PPh}_{3}$ in dichloromethane, and the subsequent addition of $\mathrm{NH}_{4} \mathrm{PF}_{6}$ in methanol produced the salts [Pt(DMAP)(L)] $\mathrm{PF}_{6}$ (12) and $\left[\mathrm{Pt}\left(\mathrm{PPh}_{3}\right)(\mathrm{L})\right] \mathrm{PF}_{6}$ (13), respectively. In a similar manner, reaction of (8) with 0.5 equiv. of bis(diphenyl-phosphino)methane $(\mathrm{dppm})$ formed the dppm-bridged binuclear dicationic salt [(L)Pt( $\mu$ dppm)Pt(L)][PF6 $]_{2}$ (14).

Keywords: $\mathrm{Pt}(\mathrm{II})$ complexes, cyclometallation, bipyridine, acetylides, bridging complexes

\footnotetext{
* Corresponding should be addressed to Prof. K. S. D. Perera, Dept. of Chemistry, Faculty of Natural Sciences, The Open University of Sri Lanka, Sri Lanka.
}

Email: ksper@ou.ac.lk

https://orcid.org/0000-0001-5917-7327

(Received 14th March 2020; Revised 20th May 2020; Accepted 27th May 2020) (C) OUSL) 


\section{Introduction}

Interest on cyclometallated complexes of the type $\left(\mathrm{C}^{\wedge} \mathrm{N}\right) \mathrm{M}$, $\left(\mathrm{N}^{\wedge} \mathrm{C}^{\wedge} \mathrm{N}\right) \mathrm{M}$ and $\left(\mathrm{C}^{\wedge} \mathrm{N}^{\wedge} \mathrm{N}\right) \mathrm{M}$ of platinum, ruthenium and iridium has risen recently as they are capable of harvesting light (Balzani \& Campagna, 2007). Cyclometallated platinum(II) complexes containing a bidentate unit $\left(\mathrm{C}^{\wedge} \mathrm{N}\right) \mathrm{Pt}$ (Nikolaeva \& Puzyk, 2013; Murphy, et al., 2012; Shafikov, 2019), tridentate units $\left(\mathrm{N}^{\wedge} \mathrm{C}^{\wedge} \mathrm{N}\right) \mathrm{Pt}$ (Murphy, et al., 2012; Colombo, et al., 2015; Williams, et al., 2003) and $\left(\mathrm{C}^{\wedge} \mathrm{N}^{\wedge} \mathrm{N}\right) \mathrm{Pt}$ (Tsai, et al., 2015; Fung, et al., 2016; Feng, et al., 2006; Shao, et al., 2008; Lu, et al., 2004; Nolan, et al., 2013) are derived from aromatic N-donor ligands which display a variety of emissive excited states, including ligand-to-metal and metal-toligand charge transfers. Phosphorescent Pt(II) complexes possess many advantages such as a wide emission colour tunability, a better stability towards photo and chemical degradation, a very large stokes shift, and long-lived luminescent excited states (Colombo, et al., 2015). The relative energy of these excited states is strongly affected by the groups attached to these ligands and the other auxiliary ligand bonded to the Pt(II) centre. Some of the basic and well-studied ligand types are given in Figure 1; I (Nikolaeva \& Puzyk, 2013; Murphy, et al., 2012); II (Nikolaeva \& Puzyk, 2013); III (Shafikov, 2019); IV (Shafikov, 2019); V (Murphy, et al., 2012; Colombo, et al., 2015; Williams, et al., 2003); VI (Fung, et al., 2016; Feng, et al., 2006; Shao, et al., 2008; Lu, et al., 2004; Nolan, et al., 2013); and VII (Tsai, et al., 2015).
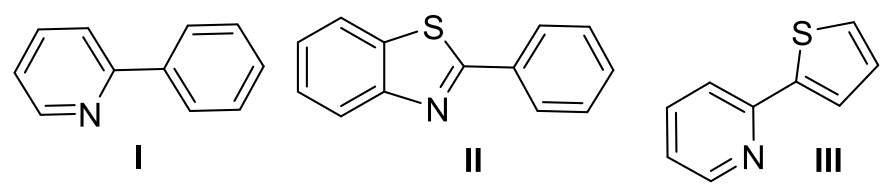<smiles>c1csc(-c2cc(-c3cccs3)ncn2)c1</smiles><smiles>c1ccc(-c2cccc(-c3ccccn3)c2)nc1</smiles><smiles>c1ccc(-c2cccc(-c3ccccn3)n2)cc1</smiles><smiles>c1ccc(-c2cccc(-c3cc[nH]n3)n2)cc1</smiles>

Figure 1. Molecular structures of ligands I - VII 
The cyclometallated platinum(II) complexes of the type $\left[\mathrm{Pt}\left(\mathrm{NH}_{3}\right)_{2}\left(\mathrm{C}^{\wedge} \mathrm{N}\right)\right] \mathrm{ClO}_{4}(\mathbf{1})$, where $\mathrm{C}^{\wedge} \mathrm{N}$ is a 2-phenylbenzothiazole ion showed intense long-lived luminescence (Figure 2) (Nikolaeva \& Puzyk, 2013). Williams et al. showed that tridentate $\left(\mathrm{N}^{\wedge} \mathrm{C}^{\wedge} \mathrm{N}\right) \mathrm{Pt}(\mathrm{II})$ complex (4) has slightly superior luminescence quantum yield when compared to that of the bidentate $\left(\mathrm{N}^{\wedge} \mathrm{C}\right) \mathrm{Pt}(\mathrm{II})$ complex (2) (Murphy, et al., 2012).

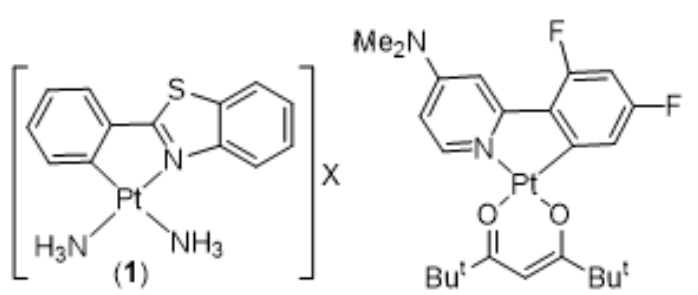

(2)
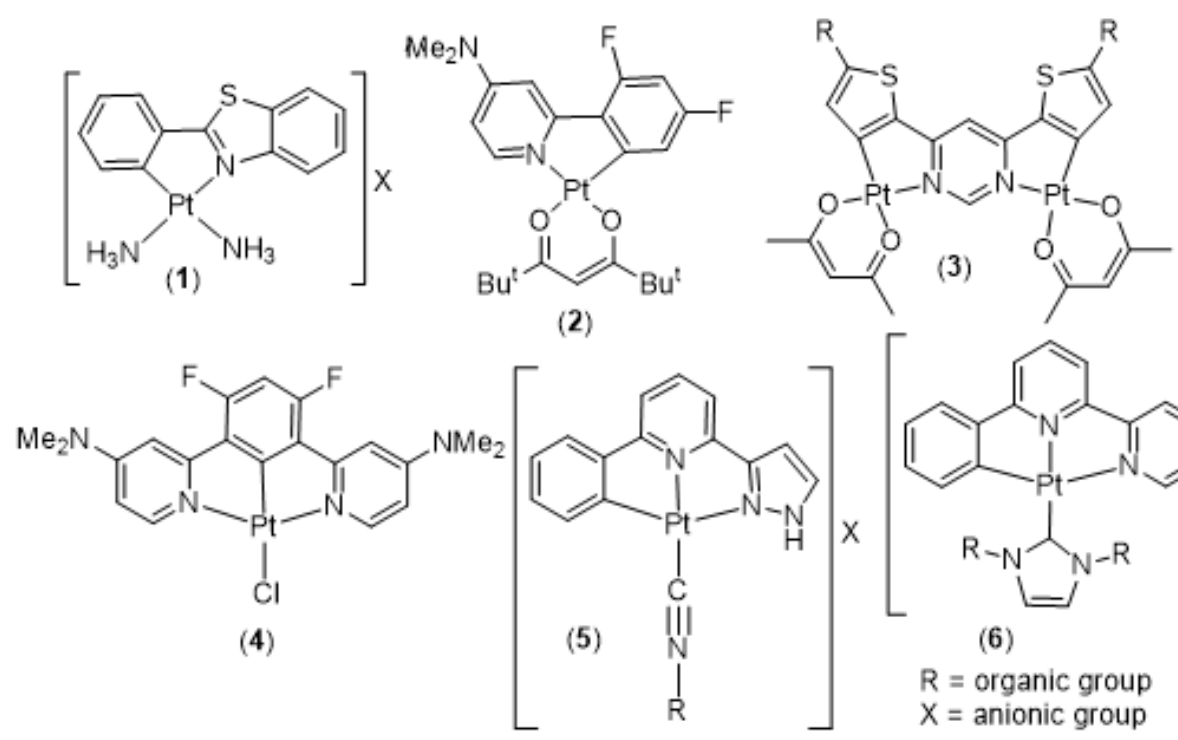

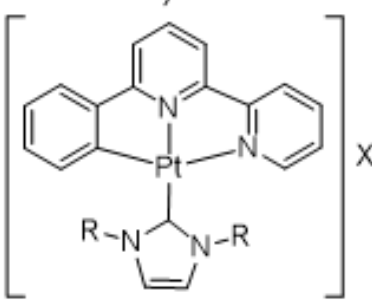

(6)

$\mathrm{R}=$ organic group

$\mathrm{X}=$ anionic group

Figure 2. Molecular structures of cyclometallated Pt(II) complexes (1) - (6)

The binuclear cyclometallated complex (3) of a substituted 4,6bis(2-thienyl)pyrimidine emits in the red region of the spectrum $\left(\lambda_{\max }=610 \mathrm{~nm}, \Phi=0.85\right.$ in deoxygenated dichloromethane at 300 $\mathrm{K})$. Colombo et al. described the synthesis of neutral tridentate $\left(\mathrm{N}^{\wedge} \mathrm{C}^{\wedge} \mathrm{N}\right) \mathrm{Pt}(\mathrm{II})$ complexes as long-lived bio-imaging probes bearing hydrophilic ethylene glycol chains to increase the water solubility and bio-compatibility. The luminescent complexes of type (5) display self-assembly and anti-cancer properties and exert cytotoxicity towards different immortalized cancer cells and primary cells (Tsai, et al., 2015). The $\mathrm{Pt}(\mathrm{II})$ complexes of the type $\left[\mathrm{Pt}\left(\mathrm{C}^{\wedge} \mathrm{N}^{\wedge} \mathrm{N}\right)(\mathrm{NHC})\right] \mathrm{O}_{3} \mathrm{SCF}_{3} \quad$ (6) $(\mathrm{NHC}=\mathrm{N}$-heterocyclic carbene) 
showed up to 15-fold higher emission intensities upon binding to mismatched DNA over matched DNA, and can be utilized for both detecting DNA abasic sites and identifying cancer cells (Fung, et al., 2016). The photo-physical and third-order nonlinear optical properties of cyclometallated (4,6-diphenyl-2,2'-bipyridyl pentynyl)platinum(II) complex have been investigated (Shao, et al., 2008). Based on their systematic studies, Lu et al. suggest that tridentate cyclometallated $\left(\mathrm{C}^{\wedge} \mathrm{N}^{\wedge} \mathrm{N}\right) \mathrm{Pt}(\mathrm{II}) \sigma$-alkynyl complexes are promising light-emitting materials, especially as phosphorescent dopants for OLED applications, due to their intense, readilyadjustable triplet emissions under ambient conditions and their thermal stability (Lu, et al., 2004).

Synthesis of the pyridyl-centred polyphenylene (7) (see Scheme 1) and its coordination chemistry particularly with rhodium and palladium have been reported (Ollangnier, et al., 2008; Perera \& Draper, 2009; Perera, 2018). It is of interest to explore the chemistry of this pyridyl-centred polyphenylene ligand (7) with platinum centres in order to prepare possible photoactive luminescent complexes of the type $\left[\left(\mathrm{C}^{\wedge} \mathrm{N}^{\wedge} \mathrm{N}\right) \mathrm{PtX}\right](\mathrm{X}=$ halide, trifluoroacetate, acetylides $),\left[\left(\mathrm{C}^{\wedge} \mathrm{N}^{\wedge} \mathrm{N}\right) \mathrm{Pt}\left(\mathrm{L}^{\prime}\right)\right] \mathrm{PF}_{6}\left(\mathrm{~L}^{\prime}=\right.$ pyridine or phosphine) and a binuclear complex containing bridging ligands. In this paper we report studies carried out to devise synthetic routes to such complexes.

\section{Methodology}

All the experiments were carried out in an inert atmosphere (dinitrogen or argon). Elemental analyses were carried out on a Carlo Erba 1006 automatic analyser. IR spectra were recorded on a PerkinElmer Spectrum One FT-IR spectrometer fitted with a universal ATR sampling accessory. Mass spectral data were obtained using a micromass LCT electrospray mass spectrometer. NMR spectra were recorded on a Bruker DPX 400 spectrometer (operating frequencies for ${ }^{1} \mathrm{H}$ and ${ }^{13} \mathrm{C}$ are 400.13 and $100.62 \mathrm{MHz}$, respectively) or Bruker Avance $\Pi 600$ spectrometer (operating frequencies for ${ }^{1} \mathrm{H}$ and ${ }^{13} \mathrm{C}$ are 600.13 and $150.9 \mathrm{MHz}$ ). ${ }^{1} \mathrm{H}$ and ${ }^{13} \mathrm{C}$ 
chemical shifts $(\delta)$ are in ppm with respect to TMS, and coupling constants $(J)$ are in $\mathrm{Hz}$. Single-crystal analyses were performed on a Bruker SMART APEX CCD diffractometer using graphite monochromised Mo- $K_{a}(\lambda=0.71073 \AA)$ radiation and refinements were obtained using SHELXS software. Figures 3 and 4 feature the images obtained by using Mercury software. 3,4,5,6-Tetraphenyl2,2'-bipyridine (7) was prepared according to a procedure recorded in relevant literature (Ollangnier, et al., 2008).

\section{[PtCl(L)] (8) and mer-[PtCl $3(\mathrm{~L})](9)$}

Suspension of the ligand (7) $(60 \mathrm{mg}, 0.13 \mathrm{mmol})$ and $\left[\mathrm{PtCl}_{2}\left(\mathrm{dmso}_{2}\right]\right.$ (55 mg, $0.13 \mathrm{mmol}$ ) in chloroform $(12 \mathrm{~mL}$ ) was refluxed for $24 \mathrm{~h}$ to give an orange precipitate. The solution was concentrated (ca. 1.5 $\mathrm{mL}$ ) and methanol (ca. $0.5 \mathrm{~mL}$ ) was added. The orange precipitate $[\mathrm{PtCl}(\mathrm{L})](8)$ was filtered off and washed with methanol (68 mg, 76\%). Upon standing, the mother liquor gave yellow crystals of mer$\left[\mathrm{PtCl}_{3}(\mathrm{~L})\right](9)$ (16 mg, 16\%).

\section{[PtCl(L)] (8)}

Found: $\mathrm{C}, \quad 58.23 ; \mathrm{H}, \quad 3.31 ; \mathrm{N}$ 3.74, calcd. (\%) for $\mathrm{C}_{34} \mathrm{H}_{23} \mathrm{ClN}_{2} \mathrm{Pt} \cdot 0.1 \mathrm{CHCl}_{3}$ : C, 58.34; $\mathrm{H}, 3.31 ; \mathrm{N}$ 3.99. IR (neat, $v, \mathrm{~cm}^{-1}$ ): 3059, 1597, 1575, 1466, 1406, 755, 727 and 701. ${ }^{1} \mathrm{H}$ NMR (400 $\left.\mathrm{MHz}, \mathrm{CDCl}_{3}\right): \delta 9.37\left(\mathrm{~m}, 1 \mathrm{H}, \mathrm{H}^{6}\right), 8.02\left(\mathrm{~d}, 1 \mathrm{H},{ }^{3} \mathrm{~J}(\mathrm{HH}) 7.8 \mathrm{~Hz}, \mathrm{H}^{8}\right)$, 7.55-7.50 (m, 2H, $\left.\mathrm{H}^{5} \& \mathrm{H}^{4}\right), 7.3-7.2\left(\mathrm{~m}, 7 \mathrm{H}, \mathrm{H}^{\mathrm{Ph}}{ }_{8} \mathrm{H}^{9}\right), 7.13-7.06(\mathrm{~m}$, $\left.4 \mathrm{H}, \mathrm{H}^{\mathrm{Ph}}\right), 6.95-6.91\left(\mathrm{~m}, 3 \mathrm{H}, \mathrm{H}^{\mathrm{Ph}}\right)$, 6.74-6.69 (m, 2H, $\left.\mathrm{H}^{\mathrm{Ph}}\right), 6.61-6.57$ $\left(\mathrm{m}, 2 \mathrm{H}, \mathrm{H}^{3}\right.$ and $\left.\mathrm{H}^{10}\right)$ and $6.02\left(\mathrm{~d}, 1 \mathrm{H},{ }^{3} \mathrm{~J}(\mathrm{HH}) 7.8 \mathrm{~Hz}, \mathrm{H}^{11}\right) .{ }^{13} \mathrm{C} \mathrm{NMR}$ was not recorded as (8) is not very soluble in most of the deuterated solvents.

\section{mer- $\left[\mathrm{PtCl}_{3}(\mathrm{~L})\right](9)$}

Found: $\mathrm{C}$, 52.65; H, 3.03; N 3.24, calcd. (\%) for $\mathrm{C}_{34} \mathrm{H}_{23} \mathrm{Cl}_{3} \mathrm{~N}_{2} \mathrm{Pt} \cdot 0.1 \mathrm{CHCl}_{3}$ : C, 52.98; H, 3.01; N 3.63. IR (neat, $v, \mathrm{~cm}^{-1}$ ): 3006, 1598, 1576, 1470, 1442, 1407, 1276, 1261, 764, 751 and 700. ${ }^{1} \mathrm{H}$ NMR $\left(400 \mathrm{MHz}, \mathrm{CDCl}_{3}\right): \delta 9.51\left(\mathrm{~m}, 1 \mathrm{H}, \mathrm{H}^{6}\right), 8.02(\mathrm{dd}, 1 \mathrm{H}$, $\left.{ }^{3} \mathrm{~J}(\mathrm{HH}) 7.5 \mathrm{~Hz},{ }^{4} \mathrm{~J}(\mathrm{HH}) 1.0 \mathrm{~Hz}, \mathrm{H}^{8}\right), 7.69-7.66\left(\mathrm{~m}, 1 \mathrm{H}, \mathrm{H}^{5}\right.$, overlaps with $\left.\mathrm{H}^{4}\right), 7.64\left(\mathrm{dt}, 1 \mathrm{H},{ }^{3} \mathrm{~J}(\mathrm{HH}) 8.0 \mathrm{~Hz},{ }^{4} \mathrm{~J}(\mathrm{HH}) 2.0 \mathrm{~Hz}, \mathrm{H}^{4}\right), 7.37-7.30$ $\left(\mathrm{m}, 6 \mathrm{H}, \mathrm{H}^{\mathrm{Ph}}\right), 7.26\left(\mathrm{dt}, 1 \mathrm{H},{ }^{3} \mathrm{~J}(\mathrm{HH}) 8.0 \mathrm{~Hz},{ }^{4} \mathrm{~J}(\mathrm{HH}) 1.5 \mathrm{~Hz}, \mathrm{H}^{9}\right), 7.17-$ $7.14\left(\mathrm{~m}, 4 \mathrm{H}, \mathrm{H}^{\mathrm{Ph}}\right), 7.00-6.97\left(\mathrm{~m}, 3 \mathrm{H}, \mathrm{H}^{\mathrm{Ph}}\right), 6.84\left(\mathrm{dd}, 1 \mathrm{H},{ }^{3} \mathrm{~J}(\mathrm{HH})\right.$ 
$\left.8.0 \mathrm{~Hz},{ }^{4} \mathrm{~J}(\mathrm{HH}) 1.0 \mathrm{~Hz}, \mathrm{H}^{3}\right), 6.77-6.70\left(\mathrm{~m}, 3 \mathrm{H}, \mathrm{H}^{\mathrm{Ph}}\right.$ and $\left.\mathrm{H}^{10}\right)$ and 6.20 $\left(\mathrm{dd}, 1 \mathrm{H},{ }^{3} \mathrm{~J}(\mathrm{HH}) 8.5 \mathrm{~Hz},{ }^{4} \mathrm{~J}(\mathrm{HH}) 1.5 \mathrm{~Hz}, \mathrm{H}^{11}\right) .{ }^{13} \mathrm{C} \mathrm{NMR}: \delta 148.6\left(\mathrm{C}^{6}\right)$, $139.0\left(\mathrm{C}^{4}\right), 132.4\left(\mathrm{C}^{9}\right), 132.1\left(\mathrm{C}^{8}\right), 130.4\left(\mathrm{C}^{11}\right), 129.3\left(\mathrm{C}^{\mathrm{Ph}}\right), 129.1$ $\left(\mathrm{CPh}^{\mathrm{Ph}}\right), 129.0(\mathrm{CPh}), 128.8\left(\mathrm{CPh}^{\mathrm{Ph}}\right), 128.5\left(\mathrm{CPh}^{\mathrm{Ph}}\right), 128.4\left(\mathrm{CPh}^{\mathrm{Ph}}\right), 128.1\left(\mathrm{C}^{3}\right)$, $127.0\left(\mathrm{CPh}^{\mathrm{Ph}}\right), 126.9\left(\mathrm{C}^{5}\right)$ and $125.3\left(\mathrm{C}^{10}\right)$.

\section{[PtCl(L)] (8)}

Suspension of the ligand (7) (60 $\mathrm{mg}, 0.13 \mathrm{mmol}),\left[\mathrm{PtCl}_{2}(\mathrm{dmso})_{2}\right]$ (55 $\mathrm{mg}, 0.13 \mathrm{mmol}$ ) and triethylamine $(36 \mathrm{mg}, 0.36 \mathrm{mmol})$ in chloroform $(12 \mathrm{~mL})$ was refluxed for $20 \mathrm{~h}$ to give an orange precipitate. The solution was concentrated (ca. $1.5 \mathrm{~mL}$ ) and the orange precipitate $[\mathrm{PtCl}(\mathrm{L})]$ was filtered off and washed with methanol (83 $\mathrm{mg}, 92 \%)$. The characterizing data as same as above.

\section{$\left[\mathrm{Pt}\left(\mathrm{O}_{2} \mathrm{CCF}_{3}\right)(\mathrm{L})\right](10)$}

Into a warm suspension of $[\mathrm{PtCl}(\mathrm{L})](8)(30 \mathrm{mg}, 0.043 \mathrm{mmol})$ in dichloromethane $(4 \mathrm{~mL})$ was added a solution of silver trifluoroacetate $\left(\mathrm{AgO}_{2} \mathrm{CCF}_{3}\right)(11 \mathrm{mg}, 0.049 \mathrm{mmol})$ in methanol (2 $\mathrm{mL}$ ). Reaction mixture was stirred at room temperature for $24 \mathrm{~h}$. The solvent was removed, and the residue was extracted with dichloromethane. The combined extract was concentrated to a low volume and methanol was added to give a red solid (31 $\mathrm{mg}, 93 \%)$. Found: $\mathrm{C}, 56.05 ; \mathrm{H}, 2.95$; $\mathrm{N} 3.25$, calcd. (\%) for $\mathrm{C}_{36} \mathrm{H}_{23} \mathrm{~F}_{3} \mathrm{~N}_{2} \mathrm{O}_{2} \mathrm{Pt}$ : C, 56.33; H, 3.02; N 3.65. IR (neat, $\mathrm{cm}^{-1}$ ): 1697, 1576, 1545, 1470, 1444, 1405, 1190,1135, 1031, 808, 784, 770, 745, 727 and 699. MS ( $\mathrm{m} / \mathrm{z}$-MALDI-TOF, $\mathrm{CH}_{2} \mathrm{Cl}_{2}$ ): found: 654.1526; calcd. 654.1509 for $\mathrm{C}_{34} \mathrm{H}_{23} \mathrm{~N}_{2} \mathrm{Pt}$, [M-O $\left.{ }_{2} \mathrm{CCF}_{3}\right]^{+}$. ${ }^{1} \mathrm{H}$ NMR $\left(400 \mathrm{MHz}, \mathrm{CD}_{3} \mathrm{CN}\right): \delta 8.81(\mathrm{br}$, $\left.\mathrm{d}, 1 \mathrm{H},{ }^{3} \mathrm{~J}(\mathrm{HH}) 4.5 \mathrm{~Hz}, \mathrm{H}^{6}\right), 7.79\left(\mathrm{t}, 1 \mathrm{H},{ }^{3} \mathrm{~J}(\mathrm{HH}) 7.8 \mathrm{~Hz}, \mathrm{H}^{4}\right), 7.68(\mathrm{~m}$, $\left.1 \mathrm{H}, \mathrm{H}^{5}\right), 7.42-7.25\left(\mathrm{~m}, 7 \mathrm{H}, \mathrm{H}^{\mathrm{Ph}}\right.$ and $\left.\mathrm{H}^{8}\right), 7.23-7.13\left(\mathrm{~m}, 4 \mathrm{H}, \mathrm{H}^{\mathrm{Ph}}\right), 7.10$ $\left(\mathrm{m}, 1 \mathrm{H}, \mathrm{H}^{9}\right), 7.01-6.91\left(\mathrm{~m}, 3 \mathrm{H}, \mathrm{H}^{\mathrm{Ph}}\right), 6.90-6.80\left(\mathrm{~m}, 2 \mathrm{H}, \mathrm{H}^{\mathrm{Ph}}\right), 6.69(\mathrm{t}$, $\left.1 \mathrm{H}, 3 \mathrm{~J}(\mathrm{HH}) 7.8 \mathrm{~Hz}, \mathrm{H}^{10}\right), 6.63\left(\mathrm{~d}, 1 \mathrm{H}, 3 \mathrm{~J}(\mathrm{HH}) 7.8 \mathrm{~Hz}, \mathrm{H}^{3}\right)$ and $5.94(\mathrm{~d}$, $\left.1 \mathrm{H},{ }^{3} \mathrm{~J}(\mathrm{HH}) 7.8 \mathrm{~Hz}, \mathrm{H}^{11}\right) .{ }^{13} \mathrm{C} \mathrm{NMR}\left(100.6 \mathrm{MHz} ; \mathrm{CD}_{3} \mathrm{CN}, \delta_{\mathrm{C}}\right): 163.6$, $157.1,156.8,152.6,150.0\left(\mathrm{C}^{6}\right), 147.9,142.0,140.4\left(\mathrm{C}^{4}\right), 136.2$, $136.1\left(\mathrm{C}^{8}\right), 136.0,135.5,135.0,133.7\left(\mathrm{C}^{9}\right), 130.7\left(\mathrm{C}^{11}\right), 129.6\left(\mathrm{CPh}^{\mathrm{Ph}}\right)$, $129.5\left(\mathrm{CPh}^{\mathrm{Ph}}\right), 129.3\left(\mathrm{C}^{\mathrm{Ph}}\right), 129.2\left(\mathrm{C}^{\mathrm{Ph}}\right), 128.8\left(\mathrm{C}^{\mathrm{Ph}}\right), 128.4\left(\mathrm{C}^{\mathrm{Ph}}\right), 128.0$ $\left(\mathrm{C}^{3 / \mathrm{Ph}}\right), 127.8\left(\mathrm{C}^{3 / \mathrm{Ph}}\right), 127.0\left(\mathrm{CPh}^{\mathrm{Ph}}\right), 126.9\left(\mathrm{C}^{5}\right)$ and $124.9\left(\mathrm{C}^{10}\right) .{ }^{19} \mathrm{~F}$ NMR (376.5 MHz, $\left.\mathrm{CDCl}_{3}\right)$ : -75.6 (s) ppm. 


\section{$\left[\mathrm{Pt}\left(\mathrm{C} \equiv \mathbf{C C}_{6} \mathrm{H}_{4} \mathrm{Bu}^{\mathrm{t}}\right)(\mathrm{L})\right](\mathbf{1 1})$}

To a degassed solution containing [PtCl(L)] (8) (25 mg, $0.036 \mathrm{mmol})$, 4-tert-butylphenyl acetylene (30 $\mathrm{mg}, 0.19 \mathrm{mmol})$ and triethyl amine $(0.5 \mathrm{~mL})$ in dichloromethane $(5 \mathrm{~mL})$, CuI $(1 \mathrm{mg}, 0.005 \mathrm{mmol})$ was added. The platinum complex went into solution gradually to give a red solution and the reaction mixture was stirred under dinitrogen for $24 \mathrm{~h}$. It was concentrated upon a low volume and methanol was added to give the required product as an orange solid (24 $\mathrm{mg}, 83 \%)$. Found: C, 67.90; H, 4.35; N 3.15, calcd. (\%) for $\mathrm{C}_{46} \mathrm{H}_{36} \mathrm{~N}_{2} \mathrm{Pt}: \mathrm{C}$, 68.05; H, 4.47; N 3.45. IR (neat, $\mathrm{cm}^{-1}$ ): 3039, 2964, 2099, 1599, $1575,1498,1467,1442,1403,1267,1107,1013,833,759,729$ and 699. MS ( $\mathrm{m} / \mathrm{z}$-MALDI-TOF, $\left.\mathrm{CH}_{2} \mathrm{Cl}_{2}\right)$ : found: 811.2547; calcd. 811.2526 for $\mathrm{C}_{46} \mathrm{H}_{36} \mathrm{~N}_{2} \mathrm{Pt},[\mathrm{M}]^{+}$. ${ }^{1} \mathrm{H}$ NMR $\left(\mathrm{CDCl}_{3}\right)$ : $\delta 9.42(\mathrm{~d}, 1 \mathrm{H}$, $\left.{ }^{3} \mathrm{~J}(\mathrm{HH}) 4.0 \mathrm{~Hz}, \mathrm{H}^{6}\right), 8.08\left(\mathrm{~d}, 1 \mathrm{H},{ }^{3} \mathrm{~J}(\mathrm{HH}) 7.0 \mathrm{~Hz},{ }^{3} \mathrm{~J}(\mathrm{PtH}) 58 \mathrm{~Hz}, \mathrm{H}^{8}\right)$, 7.65-7.47 $\left(\mathrm{m}, 3 \mathrm{H},{ }^{3} \mathrm{~J}(\mathrm{HH}) 7.8 \mathrm{~Hz}, \mathrm{H}^{\mathrm{Ar}}\right.$ and $\left.\mathrm{H}^{4}\right), 7.44-7.32(\mathrm{~m}, 3 \mathrm{H}$, $3 \mathrm{~J}(\mathrm{HH}) 7.8 \mathrm{~Hz}, \mathrm{H}^{\mathrm{Ar}}$ and $\left.\mathrm{H}^{5}\right), 7.31-7.21\left(\mathrm{~m}, 7 \mathrm{H}, \mathrm{H}^{\mathrm{Ph}}\right), 7.16-7.06(\mathrm{~m}$, $\left.3 \mathrm{H}, \mathrm{H}^{\mathrm{Ph}}\right), 7.06-6.98\left(\mathrm{~m}, 1 \mathrm{H}, \mathrm{H}^{9}\right), 6.97-6.86\left(\mathrm{~m}, 3 \mathrm{H}, \mathrm{H}^{\mathrm{Ph}}\right), 6.82-6.67$ $\left(\mathrm{m}, 2 \mathrm{H}, \mathrm{H}^{\mathrm{Ph}}\right), 6.65-6.51\left(\mathrm{~m}, 2 \mathrm{H}, \mathrm{H}^{3}\right.$ and $\left.\mathrm{H}^{10}\right), 6.09\left(\mathrm{br}, \mathrm{d}, 1 \mathrm{H},{ }^{3} \mathrm{~J}(\mathrm{HH})\right.$ $7.8 \mathrm{~Hz}, \mathrm{H}^{11}$ ) and $1.36\left(\mathrm{~s}, 9 \mathrm{H}, \mathrm{CMe}_{3}\right) .{ }^{13} \mathrm{C} \mathrm{NMR}\left(100.6 \mathrm{MHz} ; \mathrm{CDCl}_{3}\right.$, $\left.\delta_{\mathrm{C}}\right): 148.0\left(\mathrm{C}^{6}\right), 138.4\left(\mathrm{C}^{8}\right), 137.6\left(\mathrm{C}^{4}\right), 130.8\left(\mathrm{C}^{9}\right), 129.8\left(\mathrm{C}^{\mathrm{Ar}}\right), 129.7$ $\left(\mathrm{C}^{\mathrm{Ph}}\right), 129.6\left(\mathrm{C}^{\mathrm{Ph}}\right), 129.3\left(\mathrm{C}^{11}\right), 129.2\left(\mathrm{C}^{\mathrm{Ph}}\right), 128.6\left(\mathrm{C}^{\mathrm{Ph}}\right), 128.4\left(\mathrm{C}^{\mathrm{Ph}}\right)$, $127.9\left(\mathrm{CPh}^{\mathrm{Ph}}\right), 127.1\left(\mathrm{C}^{\mathrm{Ph}}\right), 126.9\left(\mathrm{C}^{3}\right), 126.8\left(\mathrm{C}^{5}\right), 126.7\left(\mathrm{C}^{\mathrm{Ph}}\right), 125.3$ $\left(\mathrm{CPh}^{\mathrm{Ph}}\right), 124.9\left(\mathrm{C}^{\mathrm{Ar}}\right), 123.1\left(\mathrm{C}^{10}\right)$ and $31.4\left(\mathrm{CMe}_{3}\right)$.

\section{[Pt(L)(DMAP)]PF ( $_{-}(12)$}

To a suspension containing [PtCl(L)] (8) $(20 \mathrm{mg}, 0.0289 \mathrm{mmol})$ and 4-dimetylaminopyridine (DMAP) $(10 \mathrm{mg}, 0.082 \mathrm{mmol})$ in chloroform $(3 \mathrm{~mL})$ a solution of $\mathrm{NH}_{4} \mathrm{PF}_{6}(12 \mathrm{mg}, 0.073 \mathrm{mmol})$ in methanol $(1 \mathrm{~mL})$ was added. The reaction mixture was refluxed for $1 \mathrm{~h}$ to give a yellow solution which was concentrated to give the required product as a yellow solid (22 mg, 81\%). Found: C, 51.91; H, 3.71; N 5.68, calcd. (\%) for $\mathrm{C}_{41} \mathrm{H}_{33} \mathrm{~N}_{4} \mathrm{PF}_{6} \mathrm{Pt} \cdot 0.25 \mathrm{CHCl}_{3}$ : C, 52.06; $\mathrm{H}, 3.52 ; \mathrm{N}$ 5.88. IR (neat, $v, \mathrm{~cm}^{-1}$ ): 2961, 1623, 1546, 1396, 835, 771 and 700. ESI-MS (acetone, $\mathrm{m} / \mathrm{z}$ ): found: 776.2342 ; calcd. 776.2353 for $\mathrm{C}_{41} \mathrm{H}_{33} \mathrm{~N}_{4} \mathrm{Pt}$, $\left[\mathrm{M}-\mathrm{PF}_{6}\right]^{+} .{ }^{1} \mathrm{H}-\mathrm{NMR}\left(400 \mathrm{MHz}, \mathrm{CDCl}_{3}, \delta\right.$ in $\left.\mathrm{ppm}\right): 8.34\left(\mathrm{~d}, 2 \mathrm{H},{ }^{3} \mathrm{~J}(\mathrm{HH})\right.$ $\left.7.0 \mathrm{~Hz},{ }^{3} \mathrm{~J}(\mathrm{PtH}) 36 \mathrm{~Hz}, \mathrm{H}^{\mathrm{Ar}}\right), 8.20\left(\mathrm{~d}, 1 \mathrm{H},{ }^{3} \mathrm{~J}(\mathrm{HH}) 4.5 \mathrm{~Hz}, \mathrm{H}^{6}\right), 7.75(\mathrm{~m}$, $\left.1 \mathrm{H},{ }^{3} \mathrm{~J}(\mathrm{HH}) 7.0 \mathrm{~Hz}, \mathrm{H}^{5}\right), 7.61\left(\mathrm{dt}, 1 \mathrm{H},{ }^{3} \mathrm{~J}(\mathrm{HH}) 8.0 \mathrm{~Hz},{ }^{4} \mathrm{~J}(\mathrm{HH}) 1.5 \mathrm{~Hz}\right.$, 
$\left.\mathrm{H}^{4}\right)$, 7.32-7.28 (m, 6H, $\left.\mathrm{H}^{\mathrm{Ph}}\right), 7.12-7.08\left(\mathrm{~m}, 4 \mathrm{H}, \mathrm{H}^{\mathrm{Ph}}\right), 7.01-6.95(\mathrm{~m}$, $\left.4 \mathrm{H}, 3 \mathrm{~J}(\mathrm{HH}) 7.5 \mathrm{~Hz}, \mathrm{H}^{\mathrm{Ph}} \& \mathrm{H}^{9}\right), 6.79\left(\mathrm{~d}, 2 \mathrm{H},{ }^{3} \mathrm{~J}(\mathrm{HH}) 7.0 \mathrm{~Hz}, \mathrm{H}^{\mathrm{Ar}}\right), 6.72-$ $6.70\left(\mathrm{~m}, 2 \mathrm{H}, \mathrm{H}^{\mathrm{Ph}}\right), 6.66-6.62\left(\mathrm{~m}, 3 \mathrm{H}, \mathrm{H}^{3}, \mathrm{H}^{8} \& \mathrm{H}^{10}\right), 6.10(\mathrm{~d}, 1 \mathrm{H}$, $\left.{ }^{3} \mathrm{~J}(\mathrm{HH}) 8.0 \mathrm{~Hz}, \mathrm{H}^{11}\right)$ and $3.25(\mathrm{~s}, 6 \mathrm{H}, \mathrm{Me}) .{ }^{13} \mathrm{C} \mathrm{NMR}(100.6 \mathrm{MHz}$; $\left.\mathrm{CDCl}_{3}, \delta_{\mathrm{C}}\right): 150.5\left(\mathrm{CAr}^{\mathrm{Ar}}\right), 149.4\left(\mathrm{C}^{6}\right), 139.4\left(\mathrm{C}^{4}\right), 132.7\left(\mathrm{C}^{8}\right), 130.7\left(\mathrm{C}^{9}\right)$, $129.8\left(\mathrm{C}^{11}\right), 129.6\left(\mathrm{CPh}^{\mathrm{Ph}}\right), 129.5\left(\mathrm{C}^{\mathrm{Ph}}\right), 129.4\left(\mathrm{C}^{\mathrm{Ph}}\right), 128.9\left(\mathrm{C}^{5}\right), 128.4$ $\left(\mathrm{C}^{\mathrm{Ph}}\right), \quad 127.6\left(\mathrm{C}^{3}\right), 127.3\left(\mathrm{CPh}^{\mathrm{Ph}}\right), 127.1\left(\mathrm{C}^{\mathrm{Ph}}\right), 124.5\left(\mathrm{C}^{10}\right), 109.4\left(\mathrm{C}^{\mathrm{Ar}}\right)$ and $39.5(M e)$.

\section{$\left[\mathrm{Pt}(\mathrm{L})\left(\mathrm{PPh}_{3}\right) \mathrm{PF}_{6}(\mathbf{1 3})\right.$}

$[\mathrm{PtCl}(\mathrm{L})](\mathbf{8})(20 \mathrm{mg}, 0.0289 \mathrm{mmol})$ and triphenylphosphine $(12 \mathrm{mg}$, $0.045 \mathrm{mmol}$ ) were heated at $60{ }^{\circ} \mathrm{C}$ in chloroform $(3 \mathrm{~mL})$ to give a yellow solution. $\mathrm{NH}_{4} \mathrm{PF}_{6}(12 \mathrm{mg}, 0.073 \mathrm{mmol})$ in methanol $(1 \mathrm{~mL})$ was then added and the solution was concentrated to give the required product as a yellow solid (29 $\mathrm{mg}, 94 \%)$. Found: C, 53.91; $\mathrm{H}, 3.34$; N 2.13, calcd. (\%) for $\mathrm{C}_{52} \mathrm{H}_{38} \mathrm{~N}_{2} \mathrm{P}_{2} \mathrm{~F}_{6} \mathrm{Pt}$ : C, 53.88; $\mathrm{H}, 3.33$; N 2.37. IR (neat, $v, \mathrm{~cm}^{-1}$ ): 3006, 1577, 1548, 1472, 1437, 1277, 1261, 833 and 750. ESI-MS (acetone, $\mathrm{m} / \mathrm{z}$ ): found: 916.2413; calcd. 916.2420 for $\mathrm{C}_{52} \mathrm{H}_{38} \mathrm{~N}_{2} \mathrm{PPt},\left[\mathrm{M}-\mathrm{PF}_{6}\right]^{+} .{ }^{31} \mathrm{P}-\mathrm{NMR}\left(161.9 \mathrm{MHz}, \mathrm{CDCl}_{3}, \delta\right.$ in ppm): 27.6 (s), ${ }^{1} \mathrm{~J}(\mathrm{PtP})=4144 \mathrm{~Hz}\left(\mathrm{PPh}_{3}\right)$ and -143.2 (septet), ${ }^{1} \mathrm{~J}(\mathrm{PF})$ $=720 \mathrm{~Hz},\left(\mathrm{PF}_{6}^{-}\right) .{ }^{1} \mathrm{H}$ NMR $\left(400 \mathrm{MHz}, \mathrm{CDCl}_{3}\right): \delta 7.90-7.86(\mathrm{~m}, 6 \mathrm{H}$, $\left.\mathrm{H}^{\mathrm{Ph}}\right), 7.62-7.57\left(\mathrm{~m}, 3 \mathrm{H}, \mathrm{H}^{\mathrm{Ph}}\right), 7.57-7.51\left(\mathrm{~m}, 4 \mathrm{H}, \mathrm{H}^{\mathrm{Ph}} \& \mathrm{H}^{4}\right), 7.31-7.27$ $\left(\mathrm{m}, 6 \mathrm{H}, \mathrm{H}^{\mathrm{Ph}}\right), 7.21-7.13\left(\mathrm{~m}, 4 \mathrm{H}, \mathrm{H}^{\mathrm{Ph}}\right), 7.01-6.94\left(\mathrm{~m}, 4 \mathrm{H}, \mathrm{H}^{\mathrm{Ph}} \& \mathrm{H}^{5}\right)$, $6.88\left(\mathrm{br}, \mathrm{d}, 1 \mathrm{H},{ }^{3} \mathrm{~J}(\mathrm{HH}) 5.6 \mathrm{~Hz}, \mathrm{H}^{6}\right), 6.82\left(\mathrm{~d}, 1 \mathrm{H},{ }^{3} \mathrm{~J}(\mathrm{HH}) 8.3 \mathrm{~Hz}, \mathrm{H}^{3}\right)$, 6.81-6.76 (m, 2H, $\left.\mathrm{H}^{\mathrm{Ph}}\right)$, 6.56-6.45 (m, 3H, $\left.\mathrm{H}^{8}, \mathrm{H}^{9} \& \mathrm{H}^{10}\right)$ and $6.28(\mathrm{~d}$, $\left.1 \mathrm{H},{ }^{3} \mathrm{~J}(\mathrm{HH}) 7.9 \mathrm{~Hz}, \mathrm{H}^{11}\right) .{ }^{13} \mathrm{C}$ NMR: $\delta 162.6,161.0,158.9,157.4$, 150.9 (C6), 150.5, 148.8, $139.7\left(\mathrm{C}^{4}\right), 138.4$ (C8), 136.3, 135.8, 135.4, 135.2, 135.1, 134.8, 132.0, 129.8 ( ( 11$), 129.7\left(\mathrm{C}^{9}\right), 129.6,129.5$, 129.3, 129.2, 129.1, 129.0, 128.8, 128.7, $128.3\left(\mathrm{C}^{3}\right), 128.2,127.1$ (C5), 126.7 and $124.6\left(\mathrm{C}^{10}\right)$.

\section{[(L)Pt(dppm)Pt(L)][PF6]2 (14)}

A suspension containing [PtCl(L)] (8) $(20 \mathrm{mg}, 0.029 \mathrm{mmol})$, bis(diphenylphosphino) methane (dppm) $(5.5 \mathrm{mg}, 0.014 \mathrm{mmol})$ and $\mathrm{NH}_{4} \mathrm{PF}_{6}(10 \mathrm{mg}, 0.061 \mathrm{mmol})$ in a mixture of degassed acetonitrile $(4 \mathrm{~mL})$ and chloroform $(1 \mathrm{~mL})$ was heated to $65^{\circ} \mathrm{C}$ for $1 \mathrm{~h}$. The resulting yellow solution was allowed to cool and was filtered. The filtrate was concentrated upon a low volume, and ethanol was 
added to give the required product as yellow needles, (24 $\mathrm{mg}, 85 \%$ ). Found: $\mathrm{C}, 54.03 ; \mathrm{H}, 3.35 ; \mathrm{N}$ 2.62, calcd. (\%) for $\mathrm{C}_{93} \mathrm{H}_{68} \mathrm{~F}_{12} \mathrm{~N}_{4} \mathrm{P}_{4} \mathrm{Pt}_{2} \cdot 1.0 \mathrm{CHCl}_{3}: \mathrm{C}, 53.69 ; \mathrm{H}, 3.31 ; \mathrm{N}$ 2.66. IR (neat, $\mathrm{U}$, $\left.\mathrm{cm}^{-1}\right)$ : 1474, 1438, 1405, 1166, 1098, 1015, 832, 770, 757, 734 and 693. MS ( $\mathrm{m} / \mathrm{z}$-MALDI-TOF, $\left.\mathrm{CH}_{2} \mathrm{Cl}_{2}\right)$ : found: 1837.3859 ; calcd. 1837.3856 for $\mathrm{C}_{93} \mathrm{H}_{68} \mathrm{~F}_{6} \mathrm{~N}_{4} \mathrm{P}_{3} \mathrm{Pt}_{2},\left[\mathrm{M}-\mathrm{PF}_{6}\right]^{+}$. ${ }^{1} \mathrm{H}$ NMR $(400 \mathrm{MHz}$, $\left.\mathrm{CD}_{3} \mathrm{CN}\right): \delta 8.12-7.91\left(\mathrm{~m}, 8 \mathrm{H}, \mathrm{H}^{\mathrm{Ph}}\right), 7.56\left(\mathrm{t}, 2 \mathrm{H}, 3 \mathrm{~J}(\mathrm{HH})=8.0 \mathrm{~Hz}, \mathrm{H}^{4}\right)$, 7.52-7.46 (m, 4H, $\left.\mathrm{H}^{\mathrm{Ph}}\right), 7.46-7.28\left(\mathrm{~m}, 22 \mathrm{H}, \mathrm{H}^{6} \& \mathrm{H}^{\mathrm{Ph}}\right), 7.21(\mathrm{~m}, 4 \mathrm{H}$, $\left.\mathrm{H}^{\mathrm{Ph}}\right), 7.14\left(\mathrm{~m}, 4 \mathrm{H}, \mathrm{H}^{\mathrm{Ph}}\right), 7.08-7.01\left(\mathrm{~m}, 6 \mathrm{H}, \mathrm{H}^{\mathrm{Ph}}\right), 6.97(\mathrm{~d}, 2 \mathrm{H}$, $\left.3 \mathrm{~J}(\mathrm{HH})=7.5 \mathrm{~Hz}, \mathrm{H}^{8}\right), 6.78\left(\mathrm{t}, 2 \mathrm{H}, 3 \mathrm{~J}(\mathrm{HH})=6.4 \mathrm{~Hz}, \mathrm{H}^{5}\right), 6.69(\mathrm{~d}, 2 \mathrm{H}$, $\left.{ }^{3} \mathrm{~J}(\mathrm{HH})=8.3 \mathrm{~Hz}, \mathrm{H}^{3}\right), 6.65\left(\mathrm{t}, 2 \mathrm{H}, 3 \mathrm{~J}(\mathrm{HH})=7.3 \mathrm{~Hz}, \mathrm{H}^{9}\right), 6.53(\mathrm{t}, 2 \mathrm{H}$, $\left.{ }^{3} \mathrm{~J}(\mathrm{HH})=7.5 \mathrm{~Hz}, \mathrm{H}^{10}\right) 6.01\left(\mathrm{~d}, 2 \mathrm{H},{ }^{3} \mathrm{~J}(\mathrm{HH})=8.0 \mathrm{~Hz}, \mathrm{H}^{11}\right)$ and $4.93-$ 4.67 (vt, $\left.4 \mathrm{H},{ }^{2} \mathrm{~J}(\mathrm{PH})=12.7 \mathrm{~Hz}, \mathrm{CH}_{2}\right) .{ }^{13} \mathrm{C} \mathrm{NMR}\left(100.6 \mathrm{MHz} ; \mathrm{CD}_{3} \mathrm{CN}\right.$, $\left.\delta_{C}\right): 160.2,158.5,157.4,152.8\left(\mathrm{C}^{6}\right), 150.9,148.8,139.5\left(\mathrm{C}^{4}\right), 138.6$, $137.8\left(\mathrm{C}^{8}\right), 136.5,136.0,136.9,135.4,135.1,133.76\left(\mathrm{C}^{\mathrm{Ph}}\right), 133.7$ $\left(\mathrm{CPh}^{\mathrm{Ph}}\right), 133.6\left(\mathrm{C}^{\mathrm{Ph}}\right), 132.1\left(\mathrm{C}^{\mathrm{Ph}}\right), 130.3\left(\mathrm{C}^{11}\right), 129.8\left(\mathrm{C}^{\mathrm{Ph}}\right), 129.5\left(\mathrm{C}^{\mathrm{Ph}}\right)$, $129.4\left(\mathrm{CPh}^{\mathrm{Ph}}\right), 129.1\left(\mathrm{C}^{9}\right), 129.0(\mathrm{CPh}), 129.0\left(\mathrm{CPh}^{\mathrm{Ph}}\right), 128.9\left(\mathrm{CPh}^{\mathrm{Ph}}\right), 128.5$ $\left(\mathrm{C}^{\mathrm{Ph}}\right), 127.9\left(\mathrm{C}^{3}\right), 127.2\left(\mathrm{CPh}^{\mathrm{Ph}}\right), 126.8\left(\mathrm{C}^{5}\right)$ and $124.8\left(\mathrm{C}^{10}\right) .{ }^{31} \mathrm{P} \mathrm{NMR}$ (161.9 MHz, $\mathrm{CD}_{3} \mathrm{CN}, \delta$ in ppm): $13.4,{ }^{1} \mathrm{~J}(\mathrm{PtP})=4208 \mathrm{~Hz}$.

\section{Results and Discussion}

Treatment of (7) with $\left[\mathrm{PtCl}_{2}(\mathrm{dmso})_{2}\right](\mathrm{dmso}=$ dimethyl sulfoxide) in boiling chloroform resulted in the formation of a mixture of cyclometallated square-planar platinum(II) complex $[\mathrm{PtCl}(\mathrm{L})](\mathbf{8})$ and an octahedral platinum(IV) complex mer-[(L)PtCl $\mathrm{Pt}_{3}$ (9), both containing an anionic tridentate $\left(\mathrm{C}^{\wedge} \mathrm{N}^{\wedge} \mathrm{N}\right)$ ligand. The orange complex (8) was not very soluble in common deuterated solvents and in the proton NMR spectrum, the proton (H6) on the carbon adjacent to nitrogen was the most deshielded and the resonance appeared as a multiplet at $9.37 \mathrm{ppm}$. The complex (9) was characterised by X-ray crystallography and confirmed the presence of mer-geometry around the platinum centre (Figure 3). In the proton NMR spectrum, the H6 proton appeared as a multiplet at $9.51 \mathrm{ppm}$. One can argue that the complex (5) could have been formed by the replacement of the platinum hydride of the intermediate $\left[\mathrm{Pt}(\mathrm{L}) \mathrm{HCl}_{2}\right]$ which was due to the oxidative addition of phenyl $\mathrm{C}-\mathrm{H}$ bond by a chloride ligand formed during the reaction 
(see Scheme 1). It is clear that the intermediate [(L) $\left.\mathrm{PtHCl}_{2}\right]$ undergoes reductive elimination of $\mathrm{HCl}$ to produce the squareplanar platinum(II) complex $[\mathrm{PtCl}(\mathrm{L})]$ (8). It is known, in the presence of a base, one could readily remove $\mathrm{HCl}$ from a platinum(IV) complex containing a $\mathrm{PtHCl}_{2}$ moiety. As expected, addition of triethylamine to the above reaction mixture improved the yield of $[\mathrm{PtCl}(\mathrm{L})](\mathbf{8})$.<smiles>c1ccc(-c2nc(-c3ccccc3)c(-c3ccccc3)c(-c3ccccc3)c2-c2ccccc2)cc1</smiles>

(7) $=\mathrm{LH}$<smiles>CC(C)CC([Mg][Mg])C(C)(C)C</smiles>

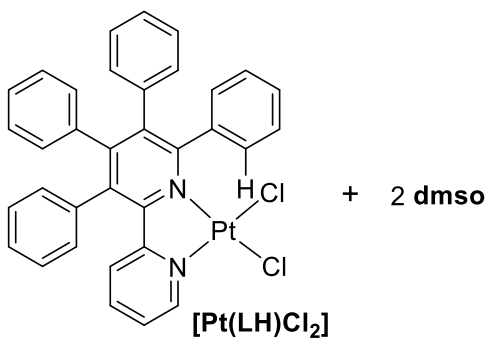
C-H activation $\checkmark$

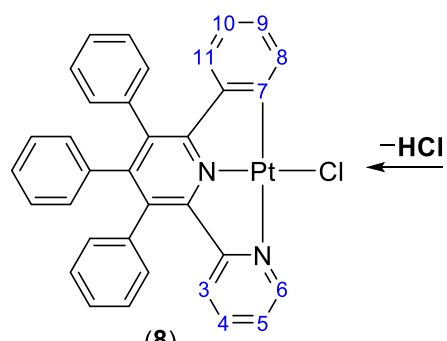

(8)

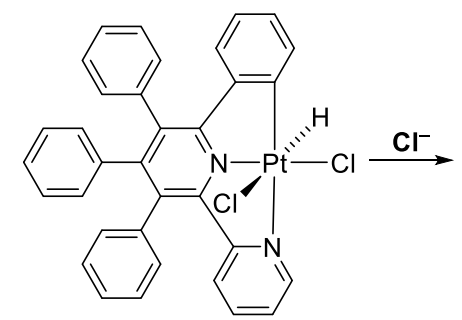

$\left[\mathrm{Pt}(\mathrm{L}) \mathrm{HCl}_{2}\right]$

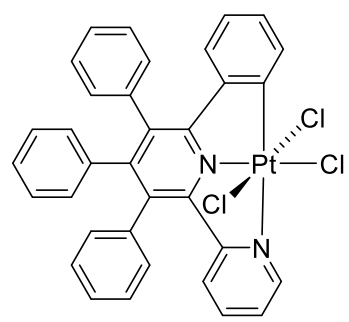

(9)

Scheme 1. Possible mechanism for the formation of complexes (8)(9); and atom labelling used for the assignment of NMR data 


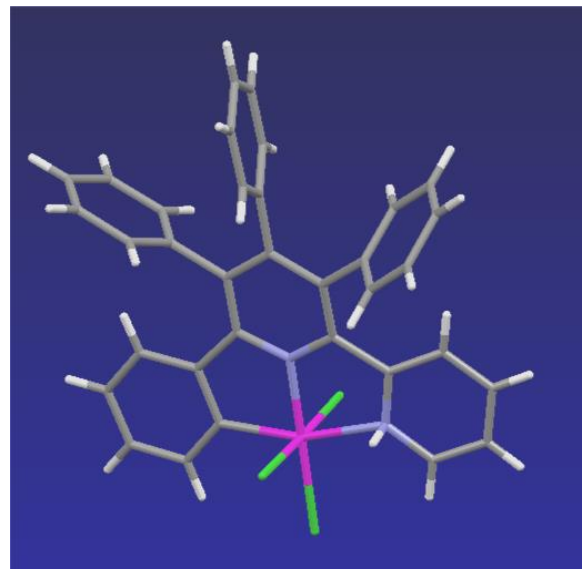

Figure 3. Molecular structure of $\operatorname{mer}-\left[(\mathrm{L}) \mathrm{PtCl}_{3}\right](\mathbf{9})$

Square-planar platinum(II) complexes are being used to study substitution reactions with anionic ligands. The chloride of $(\mathbf{8})$ can be easily replaced by trifluoroacetate ion to produce $\left[(\mathrm{L}) \mathrm{PtO}_{2} \mathrm{CCF}_{3}\right]$ (10) a red solid with 93\% yield. The IR spectrum of (10) showed an IR band at $1659 \mathrm{~cm}^{-1}$ for the $\mathrm{C}=\mathrm{O}$ group. The complex is quite soluble in organic solvents and it was well characterized. The $19 \mathrm{~F}$ NMR spectrum showed a singlet at $-75.6 \mathrm{ppm}$ for the fluorine nuclei in the $\mathrm{CF}_{3}$ group.

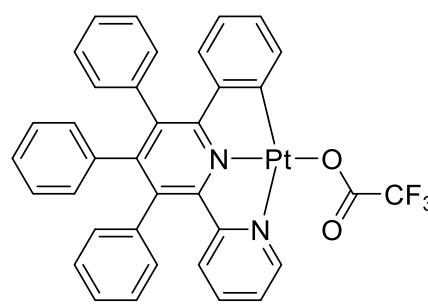

(10)

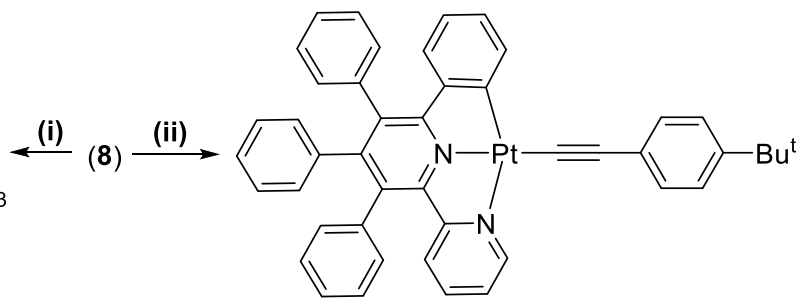

(11)

Scheme 2. Synthetic routes to complexes (10)-(11); (i) $\mathrm{AgO}_{2} \mathrm{CCF}_{3}$; (ii) $\mathrm{HC} \equiv \mathrm{CC}_{6} \mathrm{H}_{4} \mathrm{Bu}^{\mathrm{t}}-4$

Cyclometallated Pt(II) acetylides have shown interesting photophysical properties (Feng, et al., 2006; Shao, et al., 2008; Lu, et al., 2004) and the complex (8) was converted into its acetylide $\left[(\mathrm{L}) \mathrm{PtC} \equiv \mathrm{CC}_{6} \mathrm{H}_{4} \mathrm{Bu}^{\mathrm{t}-4}\right](\mathbf{1 1})$ of $83 \%$ yield, by reacting it with 4 -tert- 
butylphenyl acetylene in the presence of triethylamine and catalytic amount of CuI. In the ${ }^{1} \mathrm{H}$ NMR spectrum, the resonances of the aryl group $\left(-\mathrm{C}_{6} \mathrm{H}_{4} \mathrm{Bu}^{\mathrm{t}}-4\right)$ appeared as an $\mathrm{AB}$ pattern at 7.60 (d) and 7.40 (d) ppm with $3 \mathrm{~J}(\mathrm{HH})=7.8 \mathrm{~Hz}$ for aryl protons and a singlet at 1.36 ppm for the nine tert-butyl protons. The IR spectrum of (11) showed an IR band at $2099 \mathrm{~cm}^{-1}$ for the acetylide ligand.

It is interesting to study the substitution of the chloride ion by neutral ligands such as pyridine and phosphine ligands to make the less soluble platinum(II) complex (8) more soluble in the form of a salt. Replacement of the labile chloride ligand with 4-dimetylaminopyridine (DMAP) in the presence of $\mathrm{NH}_{4} \mathrm{PF}_{6}$ resulted in the formation of the salt $[\mathrm{Pt}(\mathrm{L})(\mathrm{DMAP})] \mathrm{PF}_{6}(\mathbf{1 2})$. The complex (12) was characterised by X-ray crystallography (Figure 4). In its ${ }^{1} \mathrm{H}$ NMR spectrum, the methyl proton resonance of $\mathrm{NMe}_{2}$ group appeared as a singlet at $3.25 \mathrm{ppm}$.

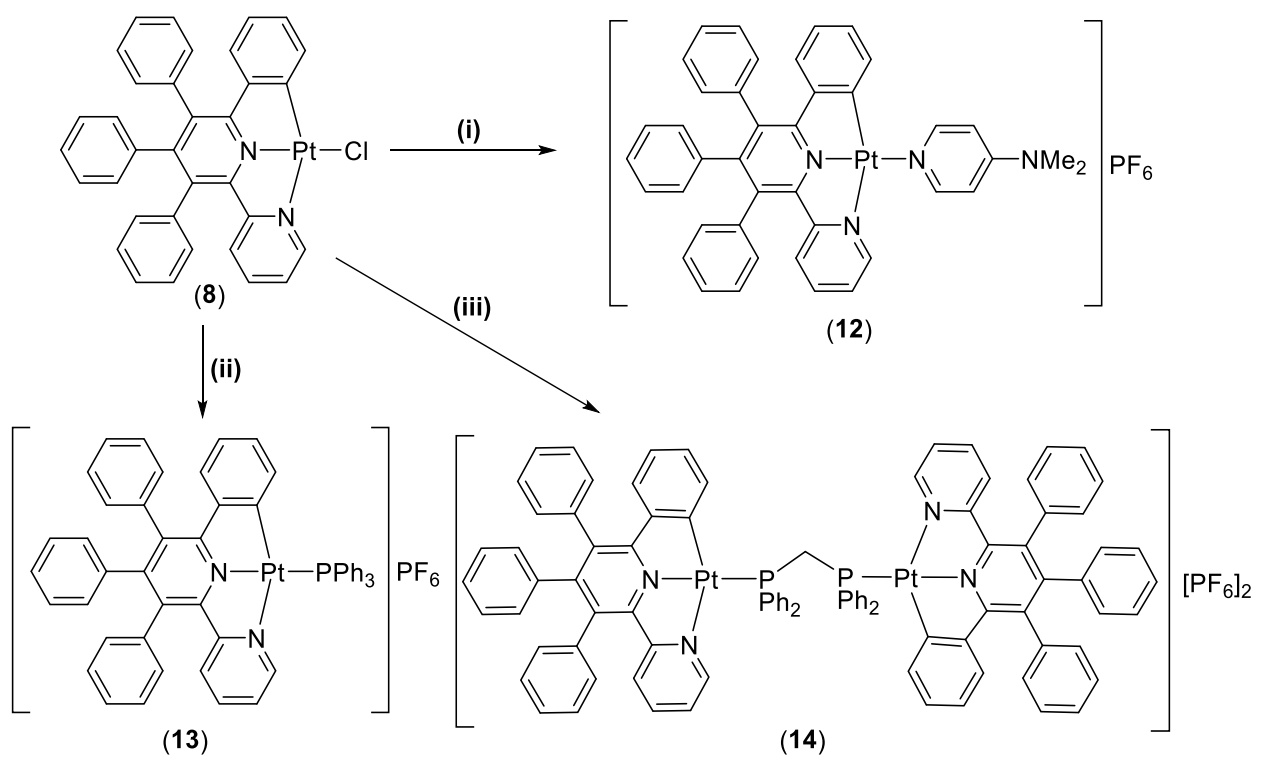

Scheme 3. Synthetic routes to complexes (12)-(14); $\mathrm{DMAP} / \mathrm{NH}_{4} \mathrm{PF}_{6}$; (i) $\mathrm{PPh}_{3} / \mathrm{NH}_{4} \mathrm{PF}_{6}$; (iii) 0.5 equiv. dppm/ $\mathrm{NH}_{4} \mathrm{PF}_{6}$; 


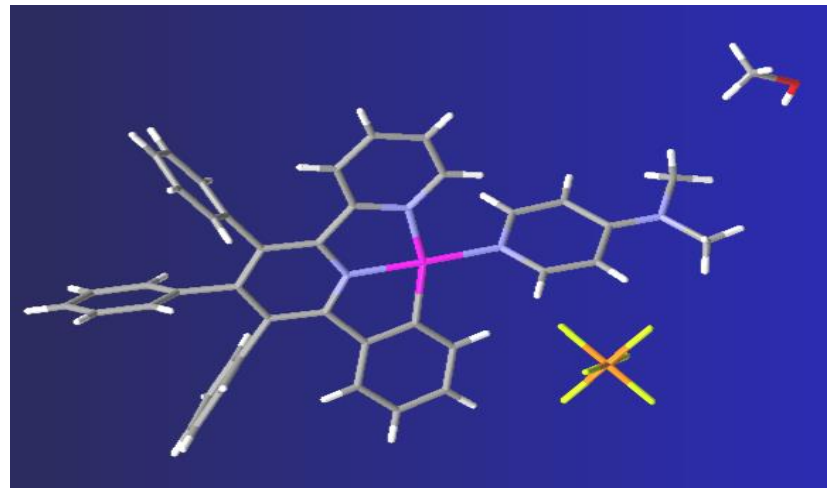

Figure 4. Molecular structure of $[\mathrm{Pt}(\mathrm{L})(\mathrm{DMAP})] \mathrm{PF}_{6}$ (12)

Replacement of the labile chloride ligand by triphenylphosphine formed the salt (13) as a yellow solid of 94\% yield. ${ }^{11} \mathrm{P}-\{1 \mathrm{H}\}$ NMR spectrum of (13) showed a singlet at $27.6 \mathrm{ppm}$ with platinum satellites, ${ }^{1} \mathrm{~J}(\mathrm{PtP})=4144 \mathrm{~Hz}$, for $\mathrm{PPh}_{3}$ and a septet at $-143.2 \mathrm{ppm}$ for the $\mathrm{PF}_{6}{ }^{-}$group. Replacement of two chloride groups from two complexes of (8) by one dppm ligand gave the dppm-bridged binuclear dicationic salt $[(\mathrm{L}) \mathrm{Pt}(\mu-\mathrm{dppm}) \mathrm{Pt}(\mathrm{L})]\left(\mathrm{PF}_{6}\right)_{2} \quad$ (14). The phosphorus resonance of (14) was $13.4 \mathrm{ppm}$ with ${ }^{1} \mathrm{~J}(\mathrm{PtP})=4208 \mathrm{~Hz}$.

\section{Conclusions}

The bulky ligand (7) containing four phenyl groups was converted into a quite insoluble cyclometallated terdentate complex $\left[\left(\mathrm{C}^{\wedge} \mathrm{N}^{\wedge} \mathrm{N}\right) \mathrm{PtCl}\right]$. However, it can be made more soluble by replacing the chloride ligand to produce the corresponding trifluoroacetate and acetylide. Introduction of a neutral ligand such as pyridine or phosphine generated more soluble salts of the type $\left[\left(\mathrm{C}^{\wedge} \mathrm{N}^{\wedge} \mathrm{N}\right) \mathrm{Pt}\left(\mathrm{L}^{\prime}\right)\right] \mathrm{PF}_{6}$ where $\mathrm{L}^{\prime}=\mathrm{PPh}_{3}$, DMAP. A binuclear complex bridging two $\left(\mathrm{C}^{\wedge} \mathrm{N}^{\wedge} \mathrm{N}\right) \mathrm{Pt}$ units with dppm was also prepared.

\section{Acknowledgements}

Author wishes to thank the Trinity College Dublin for a Research Fellowship and Professor S. M. Draper for laboratory facilities and other support. Author is also grateful Dr. John O'Brien and Dr. M. 
Ruether for recording NMR spectra; Dr Martin Feeney for providing mass spectral data; and Dr Thomas McCabe for determining crystal structures. An abstract of this study was presented at the Annual Sessions of the Institute of Chemistry, Ceylon in 2013.

\section{References}

Balzani, V. \& Campagna, S. (Ed). (2007). Photochemistry and photophysics of coordination compounds: Platinum, Top. Curr. Chem, 281, 205-268.

Colombo, A., Fiorini, F., Septiadi, D., Dragonetti, C., Nisic, F., Valore, A., Roberto, D., Mauro, M. \& De Cola, L. (2015). Neutral $\mathrm{N}^{\wedge} \mathrm{C}^{\wedge} \mathrm{N}$ terdentate luminescent $\mathrm{Pt}(\mathrm{II})$ complexes: their synthesis, photophysical properties, and bio-imaging applications, Dalton Trans., 44(18), 8478-87, DOI: 10.1039/c4dt03165b.

Feng, K., Zhang, R-Y., Wu, L-Z., Tu, B., Peng, M-L., Zhang, L-P., Zhao, D. \& Tung, C-H. (2006). Photooxidation of olefins under oxygen in platinum(II) complex-loaded mesoporous molecular sieves. J. Am. Chem. Soc. 128, 14685-14690. DOI: $10.1021 / \mathrm{ja0648256}$

Fung, S. K., Zou, T., Cao, B., Chen, T., To, W-P., Yang, C., Lok, CN. \& Che, C-M. (2016). Luminescent platinum(II) complexes with functionalized $\mathrm{N}$-heterocyclic carbene or diphosphine selectively probe mismatched and abasic DNA. Nat. Commun.7:10655 doi: 10.1038/ncomms 10655 (2016).

Lu, W., Mi, B-X., Chan, M. C. W., Hui, Z., Che, C-M., Zhu, N. \& Lee, S-T. (2004). Light-emitting tridentate cyclometallated platinum(II) complexes containing o-alkynyl auxiliaries: tuning of photo- and electrophosphorescence. J. Am. Chem. Soc. 126, 4958-4971. DOI: 10.1021/ja0317776.

Murphy, L., Brulatti, P., Fattori, V., Cocchi, M. \& Williams, J. A. G. (2012). Blue-shifting the monomer and excimer phosphorescence of tridentate cyclometallated platinum(II) complexes for optimal white-light OLEDs. Chem. Commun. 48, 5817-5819, DOI: 10.1039/C2CC31330H. 
Nikolaeva, M. V. \& Puzyk, M. V. (2013). Spectral and luminescent investigations of ammonia cyclometallated $\mathrm{Pt}(\mathrm{II})$ complexes. 114, 247-250, DOI: 10.1134/S0030400X13010219.

Nolan, D., Gil, B., Wang, L., Zhao, J. \& Draper, S. M. (2013). Structure-property relationships and ${ }^{1} \mathrm{O}_{2}$ photosensitisation in sterically encumbered diimine $\mathrm{Pt}(\mathrm{II})$ acetylide complexes. Chem. Eur. J. 19, 15615-15626. DOI: 10.1002/chem.201300759.

Ollangnier, C. M. A., Perera, S. D., Fitchett, C. M. \& Draper, S. M. (2008). Rhodium and palladium complexes of a pyridylcentred polyphenylene derivative, J. Chem. Soc. Dalton Trans. 283-290, DOI: 10.1039/b709818a.

Perera, S. D. \& Draper, S. M. (2009). Synthesis of cyclometallated Palladium(II) complexes derived from 3,4,5,6-Tetraphenyl2,2'-bipyridine. Annual Academic Sessions, The Open University of Sri Lanka.153-156

Perera, S. D. (2018). Coordination chemistry of a $\left(\mathrm{N}^{\wedge} \mathrm{N}^{\wedge} \mathrm{C}\right)$ palladacycle. Proceedings of the Open University International Research Session, (iOURS). 227-230.

Shafikov, M. Z., Daniels, R., Pander, P., Dias, F. B., Williams, J. A. G. \& Kozhevnikov, V. N. (2019). Dinuclear design of a Pt(II) complex affording highly efficient red emission: photophysical properties and application in solution processible OLEDs. ACS Appl. Mater. Interfaces. 11, 8182-8193, DOI: 10.1021 /acsami.8b18928.

Shao, P., Li, Y. \& Sun, W. (2008). Cyclometallated platinum(II) complex with strong and broadband nonlinear optical response. J. Phys. Chem. A. 112, 1172-1179. DOI: $10.1021 /$ jp710616z.

Tsai, J. L-L., Zou, T., Liu, J., Chen, T., Chan, A. O-Y., Yang, C., Lok, C-N. \& Che, C-M. (2015). Luminescent platinum(II) complexes with self-assembly and anti-cancer properties: hydrogel, $\mathrm{pH}$ dependent emission colour and sustainedrelease properties under physiological conditions. Chem. Sci., 6, 3823-3830, DOI: 10.1039/c4sc03635b. 
Williams, J. A. G., Beeby, A., Davies, E. S., Weinstein, J. A., and Wilson, C. (2003). An alternative route to highly luminescent platinum(II) complexes: cyclometallation with $\mathrm{N} \wedge \mathrm{C} \wedge \mathrm{N}-$ coordinating dipyridylbenzene ligands. Inorg. Chem. 42, 8609-8611, doi: 10.1021/ic035083+. 